\title{
OPEN LRTM effect and electronic crystal imaging on silicon surface
}

\author{
Zhong-Mei Huang ${ }^{1}$, Shi-Rong Liu ${ }^{3}$, Hong-yan Peng ${ }^{2}$, Xin Li ${ }^{1}$ \& Wei-Qi Huang ${ }^{1,2 \bowtie ~}$
}

Some interesting phenomena have been observed in the laser reflecting Talbot magnification (LRTM) effect discovered at first, in which the high-order nonlinear imaging and the plasmonic structures imaging occur. The LRTM effect images were obtained on the 1D and 2D photonic crystals fabricated by using nanosecond pulsed laser etching on silicon surface, where the high-order nonlinear imaging on the 1D and 2D photonic crystals was observed interestingly. The theory result is consistent with the experimental one, which exhibits that the suitable wave-front shape of injection beam selected in optical route can effectively enlarge the magnification rate and elevate the resolution of the Talbot image. Especially the periodic plasmonic structures on silicon surface have been observed in the LRTM effect images, which have a good application in the online detection of pulsed laser etching process. The temporary reflecting Talbot images exhibit that the electrons following with photonic frequency float on plasma surface to form electronic crystal observed on silicon at first, which is similar with the Wigner crystal structure.

The beauty and simplicity of Talbot effect images, so-called self-imaging effect, attracted scientists and resulted in numerous interesting and applications. As first, H.F.Talbot discovered the conventional Talbot effect in $1836^{1}$. Then, the effect was well understood by the Fresnel-Kichhoff diffraction theory and was explained analytically by Lord Rayleigh in $1881^{2}$. The recent developing has been made in the areas, which has attracted extensive research interesting due to the promising physical properties concerning various applications ${ }^{3-14}$. Nowadays, the new phenomena and their application in the Talbot effect have provided a new way for micro-nanostructures to be observed and analyzed by simply projecting image, involving phase components and amplitude, nonlinear images, various micro-nanoarrays images and surface plasmonic structures images ${ }^{15-31}$. In addition, the recent progress about the bi-layer structure image in the electromagnetic field was reported ${ }^{32}$. And the self-imaging effect with a similar approach in atomic ensemble EIT media has been noted as well ${ }^{33,34}$.

In the article, the LRTM effect has been discovered on one-dimensional (1D) and two-dimensional (2D) arrays on silicon surface under the illumination of Gaussian spherical wave and plane wave, which is different from the conventional Talbot effect. It provides a new way to magnify the reflecting image for observation of thicker objects with periodic structures on surface. The 1D and 2D photonic crystals on silicon surface were fabricated by using nanosecond pulsed laser etching (PLE) method, where their high-order nonlinear images were observed in the LRTM effect interestingly. Here, the resolution of the LRTM effect image was improved due to originate from the high-order nonlinear diffraction. The magnifying image of plasmonic structures in the LRTM effect was observed on silicon surface. It is interesting that the temporary reflecting Talbot images exhibit that the electrons following with photonic frequency float on plasma surface to generate electronic crystal observed at first, which is similar with the Wigner crystal structure.

LRTM effect. In the experimental results, the magnification rate of the LRTM effect image and the interval between two adjacent reflecting Talbot images could be controlled by adjusting wave-front shape of illumination to enlarge the magnification rate.

The $1 \mathrm{D}$ and 2D photonic crystal of silicon as the samples were fabricated by using nanosecond PLE method in room temperature. The laser beams at $532 \mathrm{~nm}$ or at $805 \mathrm{~nm}$ are as the foundation injection waves for illumination in the LRTM image, which are reshaped and then illuminated on the samples. In the image process, a telescope device along with inverse light path is used to obtained plane wave for illuminating samples, whose image is shown in Fig. 1a where the inset exhibits its diffraction pattern. In other way, we can tailor the wave-front shape of injection beam to get Gaussian spherical wave by adjusting beam spot in the LRTM image process, whose image is exhibited in Fig. $1 \mathrm{~b}$ where the inset shows the diffraction pattern of the 2D lattice.

${ }^{1}$ Institute of Nanophotonic Physics, Guizhou University, Guiyang 550025, China. ${ }^{2}$ Department of Physics, Hainan Normal University, Haikou 571158, China. ${ }^{3}$ State Key Laboratory of Environment Geochemistry, Institute of Geochemistry, Chinese Academy of Sciences, Guiyang 550003, China. ${ }^{\varpi}$ email: wqhuang@gzu.edu.cn 

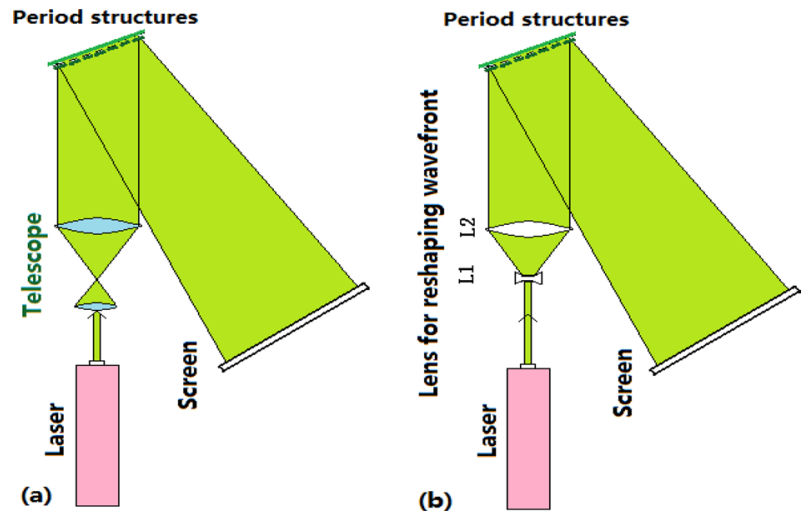

Figure 1. (a) Experimental diagram designed for reshaping Gaussian beams to plane wave, in which the telescope is used in inverse optical path. (b) Experimental diagram designed for enlarging spot of Gaussian beams, where the beam wave-front can be reshaped by adjusting lens L1 and L2 in the LRTM routing.

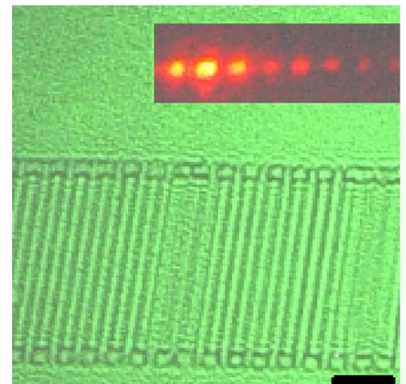

(a)

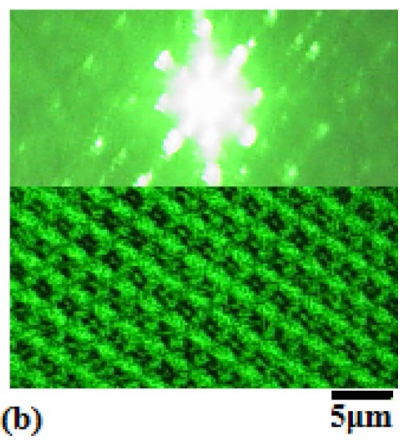

Figure 2. LRTM images on $1 \mathrm{D}(\mathbf{a})$ and $2 \mathrm{D}(\mathbf{b})$ photonic crystals of silicon under laser illumination, in which the inset windows show the space frequency images (diffraction patterns) of $1 \mathrm{D}$ and $2 \mathrm{D}$ photonic crystal structures.

\begin{tabular}{|l|c|c|c|c|c|c|c|c|c|c|}
\hline Talbot interval & 0.36 & 0.4 & 0.59 & 0.9 & 1.82 & 1.26 & 2.6 & 2.38 & 2.18 & 2.9 \\
\hline Image distance & 20.04 & 25.05 & 30.68 & 35.82 & 40.1 & 45.18 & 51 & 55.06 & 59.8 & 65.42 \\
\hline
\end{tabular}

Table 1. Relation between the interval of adjacent images and the imaging distance in the LRTM image process. Unit/mm.

Under the laser illumination, Fig. 2a shows the LRTM image on the 1D photonic crystals of silicon prepared by using PLE method, in which the inset window exhibits its diffraction pattern showing the space frequency of the $1 \mathrm{D}$ photonic crystal structure. Here, the space frequency is $1 /(2 \mu \mathrm{m})$ and the depth is about $3 \mu \mathrm{m}$. In the same way, the LRTM image is observed on the 2D photonic crystals fabricated by using PLE method on silicon, as shown in Fig. 2b, where the inset exhibits the diffraction pattern of the 2D photonic crystals of silicon.

The experimental results exhibit the relation between the interval of adjacent images so-called the Talbot interval and the imaging distance so-called the Talbot distance, as shown in Table 1, which displays an almost linear increasing evolution in the LRTM effect. The relationship between the Talbot interval and the imaging distance is shown in Fig. 3, where the red line is linear fit one and the black curve describes the experimental result in the LRTM process. In the detection, the spatial constant of periodic structure is $50 \mu \mathrm{m}$, and the laser beam at $532 \mathrm{~nm}$ is used as the Talbot beam. The linear rates can be controlled by adjusting wave-front shape for illumination. It is found that the magnifying rate of reflecting Talbot images is enlarged with increase of image distance. The magnifying rate can be enlarged by decreasing curvature radius $R_{1}$ of the wave-front, which is important for application of microscopy on reflection samples.

The Talbot effect belongs to a kind of transparent image, and the new reflecting Talbot image equals to the transformation of its optical path, in which it needs to change $\pi$ phase of propagating wave-front on surface according to the Maxwell theory on electromagnetic wave ${ }^{15,16}$. The reflecting Talbot image amplitude $\mathrm{A}(\mathrm{r}) \mathrm{can}$ be described by the formula: 


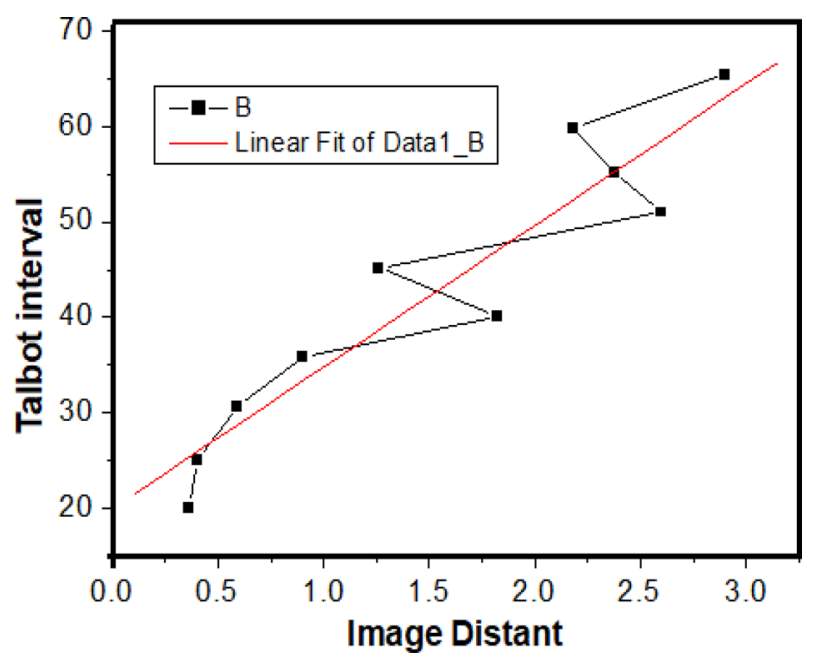

Figure 3. The curve of relationship between the Talbot interval and the imaging distance in the LRTM process, in which the black curve describes the experimental result and the red line is linear fit one.

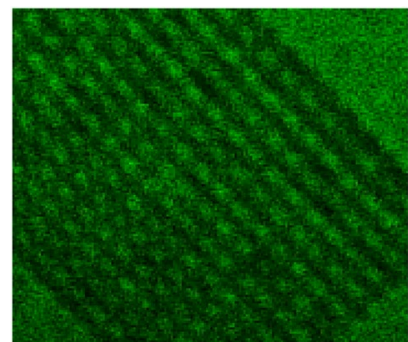

(a)

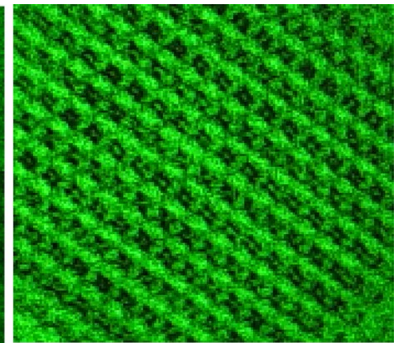

(b)

Figure 4. (a) Dark field image and (b) Bright field image, which appear at $r_{2}=r_{m}$ and $r_{2}=r_{m}+\Delta r$ respectively, where the $\Delta r$ relates to the phase term $\left[\pi\left(\lambda n^{2} R_{1} r_{2}\right) /\left(\left(R_{1}+r_{2}\right) d^{2}\right)\right]=(2 N+1) \pi$.

$$
A(r)=\sum_{n} A_{n} \exp \left[i\left(\pi \lambda n^{2} R_{1} r_{2}\right) /\left(\left(R_{1}+r_{2}\right) d^{2}\right)\right] \exp \left[-i\left(2 \pi n R_{1} r\right) /\left(\left(R_{1}+r_{2}\right) d\right)\right]
$$

In the form, $\mathrm{d}$ is the grating constant of periodic structures on surface, $\mathrm{R}_{1}$ is the curvature radius of the Gaussian wave-front and $r_{2}$ is the propagating distance from the reflection object to the observation screen. The reflecting Talbot image localizes at $r_{2}=r_{m}$ and its phase term can be described by the form:

$$
\left[\pi\left(\lambda n^{2} R_{1} r_{2}\right) /\left(\left(R_{1}+r_{2}\right) d^{2}\right)\right]=2 m \pi
$$

where the $\mathrm{m}$ is a grade number of the reflection Talbot image. Therefore, the condition for reflecting Talbot imaging is $r_{m}=m \beta /\left[1-(m \beta) / R_{1}\right]$, where $\beta$ is $\left(2 d^{2}\right) / \lambda$. It should be noted that there is $r_{m} \approx m \beta\left[1+(m \beta) / R_{1}\right]$ and the magnifying rate of the reflecting Talbot image is $M_{m}=1+r_{m} / R_{1}$, when $R_{1}>>\beta$. When the lens for magnifying in the experimental instruments are replaced by the telescope device, the $\mathrm{R}_{1}$ will turn to infinity and the distance $r_{m}$ of the reflecting Talbot imaging will be $m \beta$ related to illumination of plane wave, here the interval distance of reflecting Talbot images is almost a constant.

These results are consistent with that of experiments. It is interesting that the bright field image or the dark field image appears at $r_{2}=r_{m}$ or $r_{2}=r_{m}+\Delta r$, respectively, where the $\Delta$ r relates to the phase term $\left[\pi\left(\lambda n^{2} R_{1} r_{2}\right) /((\right.$ $\left.\left.\left.\mathrm{R}_{1}+\mathrm{r}_{2}\right) \mathrm{d}^{2}\right)\right]=(2 \mathrm{~N}+1) \pi$, as shown in Fig. $4 \mathrm{a}$ (dark field image) and in Fig. $4 \mathrm{~b}$ (bright field image). The magnifying rate of the LRTM measured in the experiments reaches to about 2000.

Nonlinear LRTM images. In the LRTM system with laser at $532 \mathrm{~nm}$, selecting higher-order diffraction and selecting nonlinear reflecting Talbot field can elevate the resolution of image through operating the aperture stop, in which the geometry details related to higher frequencies of space are obtained. Here, the nonlinear LRTM images involve the higher-order diffraction and the higher space frequencies in the reflecting Talbot field. Figures 5 and 6 show the differences between the general reflecting Talbot image and the nonlinear reflecting Talbot image on 1D and 2D photonic crystals of silicon, respectively. Figures 5a and 6a show the general LRTM images on $1 \mathrm{D}$ and 2D photonic crystals of silicon, respectively. The inset of Fig. 5a exhibits the SEM image. The 


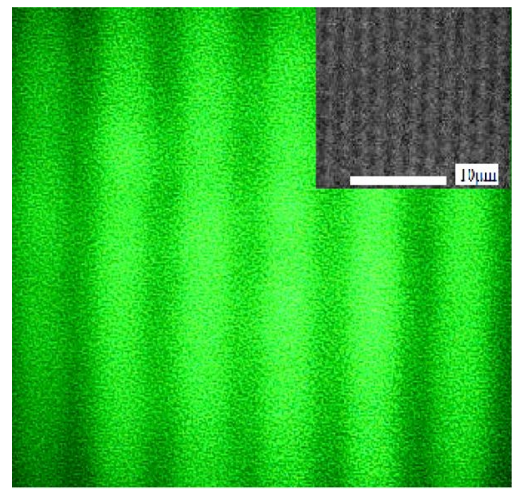

(a)

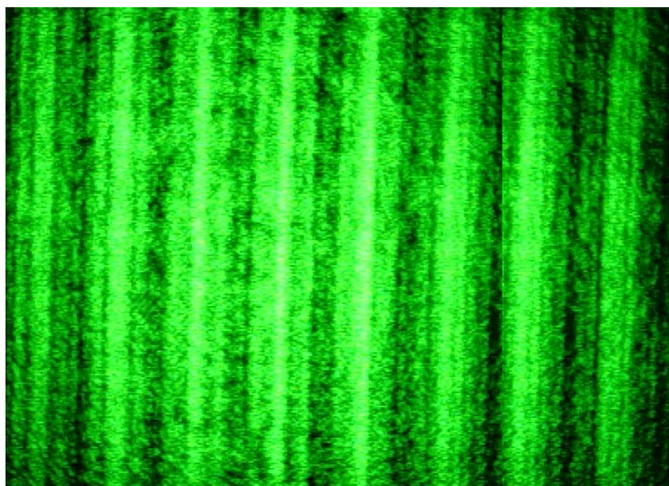

(b)

Figure 5. (a) General LRTM image, in which the inset is the SEM image; (b) nonlinear LRTM image on 1D photonic crystal of silicon.

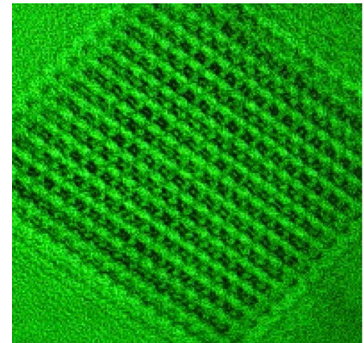

(a)

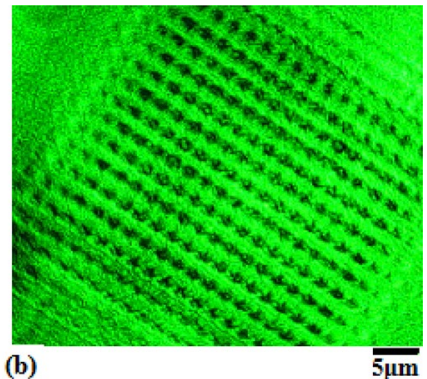

(b) $\overline{5 \mu \mathrm{m}}$

Figure 6. (a) General LRTM image and (b) nonlinear LRTM image on 2D photonic crystal of silicon.

higher order diffraction and nonlinear LRTM images on 1D and 2D photonic crystals of silicon are respectively shown in Figs. 5b and 6b, in which the geometry details can be obtained obviously.

It is interesting to make a comparison between Fig. 5a,b, in the latter image there are much more detail geometry structures in the nonlinear LRTM image on the 1D photonic crystals of silicon, which are related to higher order diffraction. In the same way to make a comparison between Fig. 6a,b, the detail geometry structures on the 2D photonic crystals of silicon are exhibited in the nonlinear LRTM image because of involving higher order diffraction.

LRTM image on plasmonic structures and electronic crystal. The plasma is produced on silicon surface by using nanosecond pulsed laser at $355 \mathrm{~nm}$, in which the plasma frequency is described by the form:

$$
\omega^{2}{ }_{p}=\mathrm{Ne}^{2} / \epsilon \mathrm{m}
$$

where $\mathrm{N}$ is electron density of plasma ${ }^{35}$. The plasmonic structures can be built on silicon surface due to resonance by coupling between photon and plasma, where the plasmon frequency is described by the formula:

$$
\omega^{2}{ }_{\mathrm{q}}=\omega_{\mathrm{p}}^{2}+(3 / 5) \mathrm{q}^{2}\left(\mathrm{hk}_{\mathrm{f}} / 2 \pi \mathrm{m}\right)^{2}
$$

$\mathrm{n}$ which $\mathrm{k}_{\mathrm{f}}$ is the Fermi vector and $\mathrm{q}$ is the quantum number of Plasmon ${ }^{36,37}$. In the experimental system, the periodic plasmonic structures take place on silicon surface, involving 1D and 2D periodic structures are detected on the Talbot screen, in which Talbot laser and lens are used respectively in the imaging process of the LRTM effect. The LRTM images of the periodic plasmonic structures on silicon surface can be exhibited on the Talbot screen.

Interestingly, the $1 \mathrm{D}$ periodic plasmonic structures are observed on silicon surface in the LRTM image as shown in Fig. 7a, and the 2D periodic plasmonic structures are observed on silicon surface in the LRTM image as shown in Fig. 7b, in which the inset circles exhibit the details of plasmonic structures.

The interaction between photons and plasmons produces surface electron gas on silicon ions background in vacuum with nanosecond pulsed laser irradiation on silicon surface, where only electrons can follow with the photons resonance with high frequency to separate the electrons for generating the electron crystal on surface ${ }^{36}$.

The temporary periodic structure of electronic crystal occurs in vacuum, where reflecting Talbot image demonstrates that the electrons play a main role in the periodic structures on silicon surface in vacuum because of only electronic resonance speeding with photons in nanosecond scale. Figure $7 \mathrm{c}$ shows the Wigner electronic crystal on the LRTM image in vacuum, in which the rectangle and hexagonal lattices of transient anomalous 


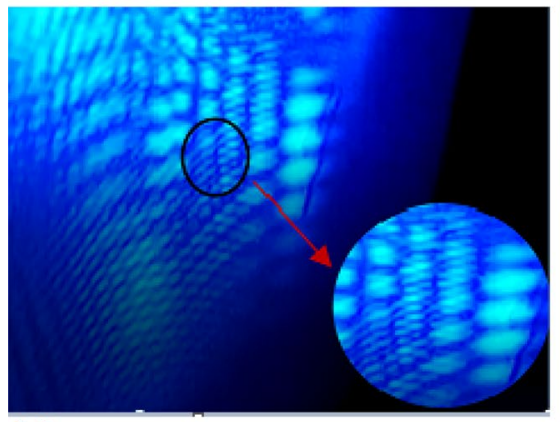

(a)

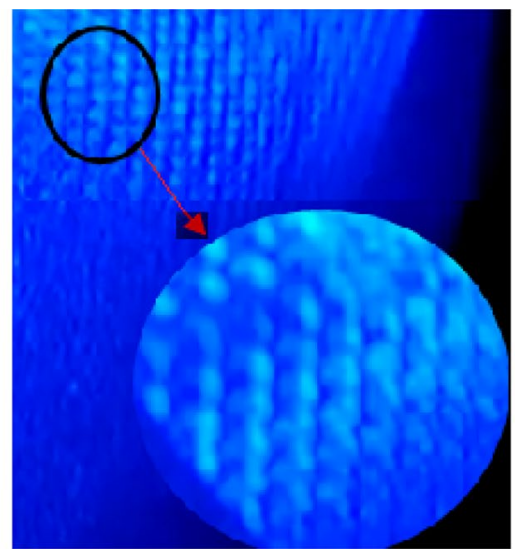

(b)
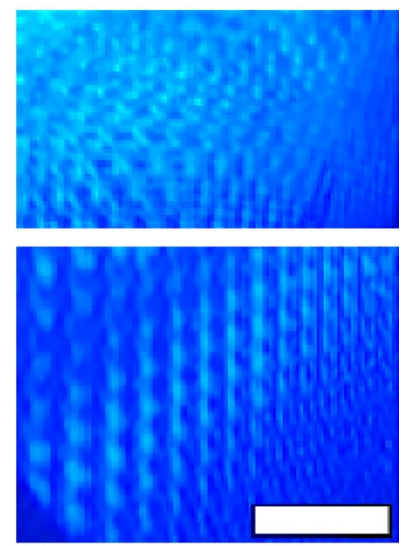

(c)

$500 \mathrm{~nm}$

Figure 7. (a) LRTM image of the 1D plasmonic periodic structures on silicon surface in the PLE process, (b) laser reflecting Talbot magnified image of the 2D plasmonic periodic structures on silicon surface in the PLE process, (c) LRTM image of the Wigner electronic crystal with the fourfold (bottom) and the sixfold (top) symmetry lattice on silicon surface in vacuum.

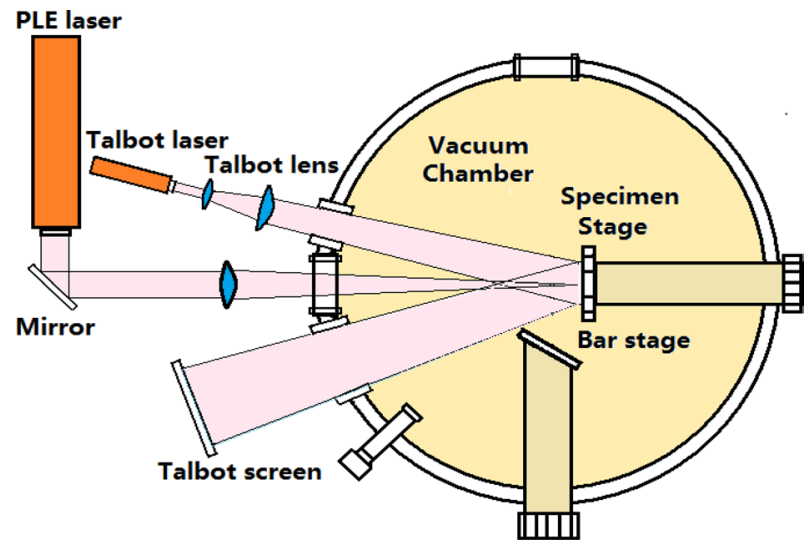

Figure 8. Diagram of the PLE cavity and the LRTM image combination device.

electron crystal have been observed on silicon surface at first. These beautiful shapes of the electron crystal structures occur in fourfold and sixfold symmetry respectively, where the size of the electron crystal cells is about $200 \mathrm{~nm}$. It is demonstrated in the decay spectra of the electronic lattice that its lifetime in vacuum is kept in nanosecond scale ${ }^{38}$.

It is interesting to make a comparison between the images of the plasmonic periodic structures and the image of the Wigner electronic crystal, as shown in Fig. 7, where the image (a) and (b) respectively exhibits the 1D and 2D plasmonic periodic structure on silicon surface in Ar gas (10 Pa), but the image (c) shows the Wigner electronic crystal on silicon surface in vacuum $\left(10^{-6} \mathrm{~Pa}\right)$. Here, the differences are very obviously: in the former images, there is not any crystal lattice in the periodic structures, but in the latter image, the two-dimensional Bravais lattices of the electron crystals occur with the fourfold and the sixfold symmetry shapes, where the Wigner electronic crystal with the rectangular lattice (bottom image) and the hexagonal lattice (top image) have obviously been observed on silicon surface in Fig. 7c.

The LRTM effect can be applied in observation on line for the fabrication in the pulsed laser etching (PLE) process, in which the PLE cavity and the LRTM image combination device are shown in Fig. 8.

In the experimental process, a silicon wafer of P-type oriented substrate is taken on the sample stage in the PLE devices, and a third harmonic of pulsed Nd:YAG laser at $355 \mathrm{~nm}$ with ns pulsed width is used to etch on $\mathrm{Si}$ surface by controlling scanning in vacuum or in different environment. The lattice structure of silicon surface on the specimen stage can be detected on the Talbot screen. In vacuum, the LRTM images of the electron crystal structures can be observed on silicon surface. And the period structures of surface plasmons on the LRTM images in various environments can be detected in the PLE process.

In summary, the LRTM effect under laser illumination has been observed on 1D and 2D photonic crystals of silicon prepared by pulsed laser etching. In the experiment it was demonstrated that the interval of reflecting Talbot images increases with increase of the image distance under laser beam. It is important for application that the magnifying rate of reflecting Talbot images can be controlled by adjusting the wave-front shape for laser illumination. In the LRTM effect, the high-order and nonlinear images have been observed obviously. It should 
be noted that selecting higher diffraction grades in nonlinear optical process can get a higher resolution in the LRTM images. It is interesting that we have observed the Wigner electronic crystal on plasma surface of silicon by using the LRTM effect image. The various periodic structures of surface plasmons on silicon can be measured in nanosecond pulsed laser etching process through the LRTM effect, which should have a good application on magnifying micro-nanostructures on line of PLE fabrication.

\section{Method}

Fabrication of 1D and 2D photonic crystals on silicon. Some silicon wafers (100) oriented substrate were taken on the sample stage in the combination fabrication system with pulsed laser etching (PLE) devices. A pulsed Nd:YAG laser (wavelength: $1064 \mathrm{~nm}$, pulse length: $60 \mathrm{~ns}$ FWHM, repetition rate: 1000) was used to etch the $1 \mathrm{D}$ photonic crystal by line-scanning way and the $2 \mathrm{D}$ photonic crystal by point-scanning way on silicon surface. The nanosecond pulsed laser is focused on sample surface, whose spot diameter is in submicron scale. The frame construction of the PLE cavity and the LRTM image combination device is shown in Fig. 8.

Received: 30 September 2020; Accepted: 10 February 2021

Published online: 16 April 2021

\section{References}

1. Talbot, H. F. Facts relating to optical science. Philos. Mag. 9, 401. https://doi.org/10.1080/14786443408648329 (1836).

2. Rayleigh, L. On copying diffraction-gratings, and on some phenomena connected therewith. Philos. Mag. 11, 196. https://doi.org/ $10.1080 / 14786448108626995$ (1881).

3. Patorski, K. The self-imaging phenomenon and its applications. Prog. Opt. 27, 1-108. https://doi.org/10.1117/12.498261 (1989).

4. David, C., Nöhammer, B., Solak, H. H. \& Ziegler, E. Differential x-ray phase contrast imaging using a shearing interferometer. Appl. Phys. Lett. 81, 3287. https://doi.org/10.1063/1.1516611 (2002).

5. Wen, J. M., Du, S. W., Chen, H. Y. \& Xiao, M. Direct measurement of versatile surface plasmon polaritons excited by split polarization. Appl. Phys. Lett. 98, 081108. https://doi.org/10.1063/1.3552673 (2011).

6. Maddaloni, P. et al. Mid-infrared tunable two-dimensional Talbot array illuminator. Appl. Phys. Lett. 94, 121105. https://doi.org/ $10.1063 / 1.3109794(2009)$.

7. David, P. et al. Narrow-line coherently combined tapered laser diodes in a Talbot external cavity with a volume Bragg grating. Appl. Phys. Lett. 93, 211102. https://doi.org/10.1063/1.3036896 (2008).

8. Maram, R., Howe, J. V., Li, M. \& Azaña, J. Noiseless intensity amplification of repetitive signals by coherent addition using the temporal Talbot effect. Nat. Commun. 5, 5163. https://doi.org/10.1038/ncomms6163 (2014).

9. Zhang, Y., Wen, J. M., Zhu, S. N. \& Xiao, M. Nonlinear Talbot effect. Phys. Rev. Lett. 104, 183901. https://doi.org/10.1103/PhysR evLett.104.183901 (2010).

10. Sanza, A. S. \& Miret-Artés, S. A causal look into the quantum Talbot effect. J. Chem. Phys. 126, 234106. https://doi.org/10.1063/1. 2741555 (2007).

11. Joseph, M. L., Leaird Daniel, E. \& Weiner Andrew, M. A temporal cloak at telecommunication data rate. Nature 498, 205. https:// doi.org/10.1038/nature12224 (2013).

12. Kiyohara, J. et al. Development of the Talbot-Lau interferometry system available for clinical use. AIP Conf. Proc. 1466, 97-102. https://doi.org/10.1063/1.4742275 (2012).

13. Teng, S., Wang, J., Zhang, W. \& Cui, Y. Talbot effect of the defective grating in deep Fresnel region. Opt. Commun. 336, $213-223$. https://doi.org/10.1016/j.optcom.2014.10.013 (2015).

14. Kim, M. S., Scharf, T., Mühlig, S., Rockstuhl, C. \& Herzig, H. P. Engineering photonic nanojets. Opt. Express 19, 10206-10220. https://doi.org/10.1364/OE.19.010206 (2011).

15. Li, W. L., Li, H. Y., Gao, B. \& Yu, Y. T. Investigation on the plasmon Talbot effect of finite-sized periodic arrays of metallic nanoapertures. Sci. Rep. 7, 45573. https://doi.org/10.1038/srep45573 (2017).

16. Li, K. et al. Discrete Talbot effect in dielectric graphene plasmonic waveguide arrays. Carbon 118, 192-199. https://doi.org/10. 1016/j.carbon.2017.03.047 (2017).

17. de Chatellus, H. G., Cortés, L. R., Deville, A., Seghilani, M. \& Azaña, J. Diffraction-induced bidimensional Talbot self-imaging with full independent period control. Phys. Rev. Lett. 118, 133903. https://doi.org/10.1103/PhysRevLett.118.133903 (2017).

18. Maram, R., Cortés, L. R., Van Howe, J. \& Azaña, J. Energy-preserving arbitrary repetition-rate control of periodic pulse trains using temporal Talbot effects. J. Lightwave Technol. 4, 658. https://doi.org/10.1109/JLT.2017.2648511 (2017).

19. Mondal, P. et al. Experimental realization of Talbot array illumination for a 2-dimensional phase grating. J. Appl. Phys. 120, 153103. https://doi.org/10.1063/1.4965696 (2016).

20. Guan, B. O., Ran, Y., Feng, F. R. \& Jin, L. Formation and applications of the secondary fiber bragg grating. Sensors 17, 398. https:// doi.org/10.3390/s17020398 (2017).

21. Wang, X. F., Lewis, E. \& Wang, P. F. Investigation of the self-imaging position of a single mode-multimode-singlemode optical fiber structure. Microwave Opt. Technol. Lett. 59, 1645-1651. https://doi.org/10.1002/mop.30605 (2017).

22. Aymerich, M., Nieto, D., Álvarez, E. \& Flores-Arias, M. T. Laser surface microstructuring of biocompatible materials using a microlens array and the Talbot effect: Evaluation of the cell adhesion. Materials 10, 214. https://doi.org/10.3390/ma10020214 (2017).

23. Santra, B. et al. Measuring finite-range phase coherence in an optical lattice using Talbot interferometry. Nat. Commun. 8, 15601. https://doi.org/10.1038/ncomms15601 (2017).

24. Udem, T. \& Ozawa, A. Mode locking based on the temporal Talbot effect. Appl. Phys. B 123, 100. https://doi.org/10.1007/s00340017-6676-0 (2017).

25. Sun, Y. Y. \& Pang, S. Multi-perspective scanning microscope based on Talbot effect. Appl. Phys. Lett. 108, 021102. https://doi.org/ $10.1063 / 1.4939873(2016)$.

26. Wang, L. C. et al. Nanostructure nitride light emitting diodes via the Talbot effect using improved colloidal photolithography. Nanoscale 9, 7021. https://doi.org/10.1039/C7NR01586K (2017).

27. Li, C. et al. Optical Talbot carpet with atomic density gratings obtained by standing-wave manipulation. Phys. Rev. A 95, 033821 . https://doi.org/10.1103/PhysRevA.95.033821 (2017).

28. Wang, X. Y. et al. Self-focusing and the Talbot effect in conformal transformation optics. Phys. Rev. Lett. 119, 033902. https://doi. org/10.1103/PhysRevLett.119.033902 (2017).

29. Walls Jamie, D. \& Daniel, H. The Talbot effect for two-dimensional massless Dirac fermions. Sci. Rep. 6, 26698. https://doi.org/10. $1038 /$ srep26698 (2016)

30. Wen, F. et al. Two-dimensional Talbot self-imaging via electromagnetically induced lattice. Sci. Rep. 7, 41790. https://doi.org/10. 1038/srep41790 (2017). 
31. Wen, J., Zhang, Y. \& Xiao, M. The Talbot effect: Recent advances in classical optics, nonlinear optics, and quantum optics. Adv. Opt. Photon. 5, 83-130 (2013).

32. Jiang, R. et al. Colloidal gold nanocups with orientation-dependent plasmonic properties. Sci. Adv. 2, 1501168. https://doi.org/10. 1002/adma.201601442 (2016).

33. Wen, F. et al. Efficient and tunable photoinduced honeycomb lattice in an atomic ensemble. Laser Photon. Rev. 12, 1800050. https:// doi.org/10.1002/lpor.201800050 (2018).

34. Wen, F. et al. Optically induced atomic lattice with tunablenear-field and far-field diffraction patterns. Photon. Res. 5, 676. https:// doi.org/10.1364/PRJ.5.000676 (2017).

35. Huang, W. Q., Xu, L., Wu, K. Y. \& Liu, S. R. Enhancement of photoluminescence emission in low-dimensional structures formed by irradiation of laser. J. Appl. Phys. 102, 053517 (2007).

36. Huang, W. Q., Huang, Z. M., Miao, X. J., Liu, S. R. \& Qin, C. J. Plasmonic emission and plasma lattice structures induced by pulsed laser in Purcell cavity on silicon. Chin. Phys. B 24, 104209. https://doi.org/10.1088/1674-1056/24/10/104209 (2015).

37. Li, L. L. \& Xu, W. Surface plasmon polaritons in a topological insulator embedded in an optical cavity. Appl. Phys. Lett. 104, 111603 (2014).

38. Wigner, E. Effects of the electron interaction on the energy levels of electrons in metals. Trans. Faraday Soc. 34, 678. https://doi. org/10.1039/TF9383400678 (1938).

\section{Acknowledgements}

Support from the National Key Laboratory of Surface Physics in Fudan University, from the National Natural Science Foundation of China (NSFC) (11847084), from the Science Foundation of Guizhou (No. 20185781) and the Science Foundation of Guizhou university (Nos. 2017(03), 20185781-17) are gratefully acknowledged.

\section{Author contributions}

Z.-M.H. is a main researcher in investigation work, who is the main researchers in experimental work, took part in the preparing process of the samples, took part in preparing Figs. 4, 5, 6 and made spectra measurement on the samples; S.-R.L. is a main researcher in experimental work, who took part in preparing Figs. 2, 3, 4 and took part in measurement on the samples; H.-Y. Peng is a main researcher in experimental work, who took part in preparing Figs. 3, 4 and took part in measurement on the samples; X.L. is a main researcher in experimental work, who took part in measurement on the samples; W.-Q.H. is the main writers and researchers, who wrote the main manuscript text and prepared Figs. 1, 2, 3, 4, 5, 6, 7, 8, provided new ideas and designed investigation plan in research. All authors discussed the results and commented on the paper.

\section{Competing interests}

The authors declare no competing interests.

\section{Additional information}

Correspondence and requests for materials should be addressed to W.-Q.H.

Reprints and permissions information is available at www.nature.com/reprints.

Publisher's note Springer Nature remains neutral with regard to jurisdictional claims in published maps and institutional affiliations.

(c) (i) Open Access This article is licensed under a Creative Commons Attribution 4.0 International c. License, which permits use, sharing, adaptation, distribution and reproduction in any medium or format, as long as you give appropriate credit to the original author(s) and the source, provide a link to the Creative Commons licence, and indicate if changes were made. The images or other third party material in this article are included in the article's Creative Commons licence, unless indicated otherwise in a credit line to the material. If material is not included in the article's Creative Commons licence and your intended use is not permitted by statutory regulation or exceeds the permitted use, you will need to obtain permission directly from the copyright holder. To view a copy of this licence, visit http://creativecommons.org/licenses/by/4.0/.

(C) The Author(s) 2021 